\title{
Calculation of a distribution free estimate of effect size and confidence intervals using VBA/Excel
}

\author{
Joachim Goedhart
}

Swammerdam Institute for Life Sciences, Section of Molecular Cytology, van Leeuwenhoek Centre for Advanced Microscopy, University of Amsterdam, Amsterdam, The Netherlands

Correspondence: j.goedhart@uva.nl, @joachimgoedhart

\begin{abstract}
Reporting effect sizes aids the transparent presentation and independent interpretation of scientific data. However, calculation and reporting of effect sizes for data obtained in basic research is rare. A standardized effect size was reported by Norman Cliff, known as Cliff's delta. It has several advantageous features, as (i) it makes no assumption on the shape of the underlying distribution, (ii) it works well for small to moderate samples $(n>10)$, (iii) it is easy to calculate, and (iv) its basis is readily understood by non statisticians. Here, a VBA macro, implemented in Excel, is presented. The macro takes two independent samples as input and calculates Cliff's delta with 95\% confidence intervals. The macro will reduce the barrier for calculating the effect size and can be a valuable tool for research and teaching.
\end{abstract}

\section{Introduction}

The use of Null Hypothesis Significance Testing (NHST) for evaluation of scientific data has been highly debated (Goodman, 2008; Cumming, 2014; Nuzzo, 2014). Several papers have highlighted misinterpretation of NHST and resulting p-values (Goodman, 2008; Halsey et al., 2015; Ivarsson et al., 2015; Wasserstein and Lazar, 2016) and have called for use of estimation statistics as alternative (Nakagawa and Cuthill, 2007; Cumming, 2014; Claridge-Chang and Assam, 2016) or additional (Drummond and Tom, 2012; Sullivan and Feinn, 2012) strategy for data analysis and presentation. Here, I only treat the case in which the data is obtained from a randomized experiment on two independent groups. The NHST returns a p-value that indicates the probability that the data from the two groups is identical, i.e. the null hypothesis is true, given the observed data or more extreme values. If the p-value is below a predefined, arbitrary threshold, usually $p<0.05$, the result is explained as evidence in favor of an alternative hypothesis, 
with smaller $p$-values taken as stronger evidence in favor of the alternative hypothesis. Importantly, $\mathrm{p}$-values do not signify the strength of evidence in favor of an alternative hypothesis (Goodman, 2008; Schneider, 2015). Moreover, NHST and the resulting p-value do not give any information on the magnitude of the difference (Nakagawa and Cuthill, 2007; Cumming, 2014; Ivarsson et al., 2015; Motulsky, 2015). To obtain information on the magnitude of the difference or the size of an effect, the effect size needs to be calculated (Sullivan and Feinn, 2012; Cumming, 2014). The effect size is arguably the parameter that is of interest, since it is related to the biological (or clinical) phenomenon that is studied (Nakagawa and Cuthill, 2007). Nevertheless, estimation statistics is rarely used in basic research and reporting NHST dominates (Tressoldi et al., 2013).

Correct calculation of effect sizes for data that deviates from the normal distribution is rare. To enable wide utilization of effects sizes in basic research, I draw attention to a standardized effect size known as Cliff's delta, that does not make assumptions on the underlying distribution (Cliff, 1993, 1996; Vargha and Delaney, 2000). The Cliff's delta was originally derived to measure effect size on ordinal data, often encountered in psychology. It works equally well for data consisting of quantitative, continuous variables, which is the predominant output in basic research (Vargha and Delaney, 2000; Hsu, 2004). Of note, Cliff's delta is a linear transformation of the A value reported by Vargha and Delaney (Vargha and Delaney, 2000). Both effect sizes were shown to be particularly robust in case of small to moderate (10-50) sample sizes with a non-normal distribution (Delaney and Vargha, 2002; Feng and Cliff, 2004; Li, 2015).

The calculation of Cliff's delta involves the comparison of all values from dataset $A$ with that of dataset $B$. When a value from set $A$ is larger than that of set $B+1$ is noted and in the reverse situation -1 is noted. In case of ties 0 is noted. The comparison of set $A$ and $B$ can be graphically represented in a dominance matrix (Cliff, 1993), see figure 1B for an example. Summing all the noted values and dividing through the total number of counts $\left(n_{A} n_{B}\right)$ yields Cliff's delta. Negative values indicate that $B$ dominates over $A$ and positive values show that $A$ dominates $B$. Cliff's delta can also be calculated from the common Mann-Whitney U statistic (Cliff's delta $=2 U / n_{A} n_{B}-1$ ).

The absolute value of Cliff's delta ranges from 0, i.e. no effect, to 1.0, indicating a maximal effect. Since the effect is standardized, it is possible to discern different categories. Based on the categories first defined by Cohen, Vargha and Delaney (2000) calculated that Cliff's 
$\mathrm{d}$ effect sizes of $0.11,0.28$ and 0.43 correspond to small, medium and large effects respectively. These categories may serve as rough guidelines for interpreting effect sizes and should not be taken as strict rules, since the effect size should be interpreted and judged in the full context of the experiment (Nakagawa and Cuthill, 2007; Cumming, 2014). Cliff's delta has several advantageous features (Cliff, 1996; Vargha and Delaney, 2000; Hsu, 2004; Ruscio, 2008) and its most powerful aspect is the straightforward calculation and the intuitive interpretation, which can be aided by a graphical representation of the dominance matrix (Cliff, 1993). Moreover, Cliff's delta (i) needs no assumption on the underlying distribution, (ii) is robust in case of outliers or skewed distributions and performs well for normally distributed data (iii) allows comparison for samples with unequal sample size and (iv) works well for small to moderate sample sizes $(n>10)$.

\section{Rationale and development of the macro}

Several options to calculate Cliff's delta and confidence intervals $(\mathrm{Cl})$ can be found on the web. These require specialized statistics packages such as $\mathrm{R}$ (http://cran.r-project.org/web/packages/orddom/index.html) or SAS (http://citeseerx.ist.psu.edu/viewdoc/summary?doi=10.1.1.488.2246). To allow for a broader adoption of Cliff's delta, I have developed a visual basic macro that runs in Excel. The macro has been tested and shown to work using Mac OS 10.9.5 with Microsoft Excel 2011 (version 14.6.0) and using Windows 7 with Microsoft Excel 2010 (version 14.0.7163.5000). It takes two datasets as input in column A and B and calculates Cliff's delta and the asymmetric $95 \% \mathrm{Cl}$ around the point estimate using equation 5 (Feng and Cliff 2004). In addition, it presents the dominance matrix on a separate, second sheet. A third sheet presents several parameters that are used to calculate the consistent estimate of the variance and the unbiased estimate of the variance (Cliff, 1993). The $95 \% \mathrm{Cl}$ derived from the two variances are also listed.

\section{Results}

The macro is applied to a dataset previously published on the effect of several protein variants on RhoGTPase activity in single cells and the results are summarized in figure 1. The RhoGTPase activity is measured with a FRET biosensor and yields a YFP/CFP ratio value for individual cells, where a higher ratio correlates with higher activity. In one specific 
condition, we examined the effect of p63RhoGEF versus a truncated variant, $\mathrm{DH}$ in Hek293 cells. The data was first reported by van Unen in supplemental figure S2 (van Unen et al., 2015) and analyzed with NHST resulting in a p-value of 0.015 (two-tailed Mann-Whitney test). Here, the individual data points of that dataset are depicted and a boxplot (Spitzer et al., 2014) is used to summarize the data (figure 1A). It can be inferred from the figure that both datasets have a non-normal distribution and contain some extreme values, arguing against calculation of an effect size that assume a normal distribution. Calculating the Cliff's delta results in a value of $-0.32[-0.05,-0.55]$. The effect can also be appreciated by inspection of the dominance matrix (figure 1B), showing that $\mathrm{DH}$ values are generally higher that those for p63RhoGEF. A graphical representation of Cliff's delta and the $95 \%$ confidence interval is depicted in figure $1 \mathrm{C}$.

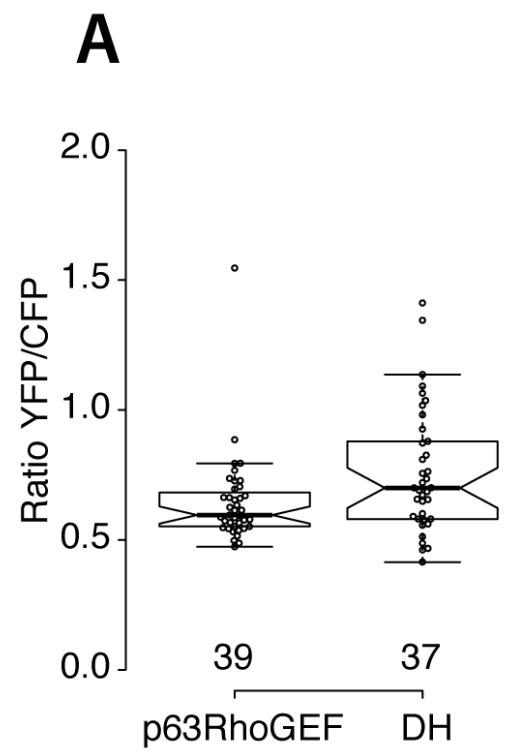

B

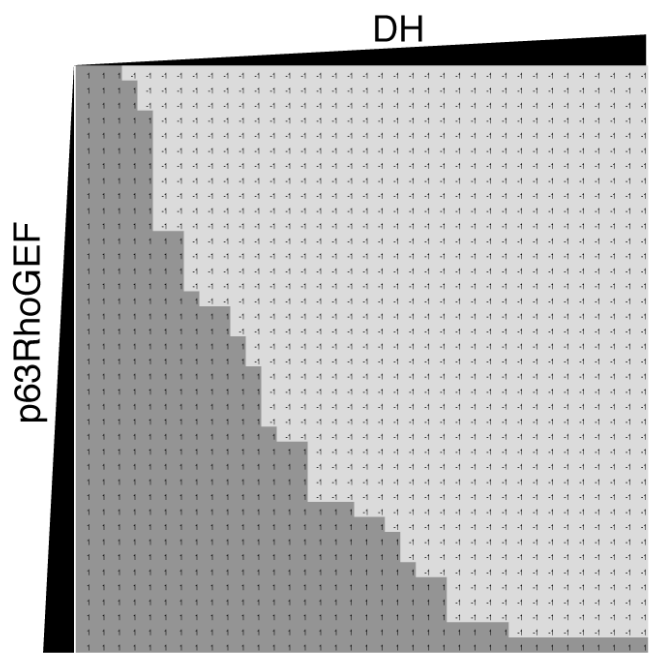

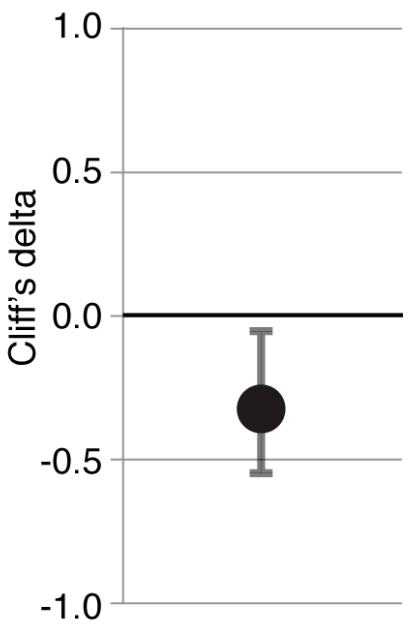

Figure 1. Example of the calculation of the dominance matrix and Cliff's delta.

(A) The individual data points shown as open circles indicate the Ratio YFP/CFP, which represents the RhoGTPase activity. The values are shown in a box plot for two conditions, p63RhoGEF and DH. The centerlines show the medians and the box limits indicate the 25th and 75th percentiles. The notches represent the $95 \%$ confidence interval for each median. The whiskers extend 1.5 times the interquartile range from the 25 th and 75 th percentiles.

(B) The dominance matrix, which is generated by the macro. All values from a dataset are sorted from low to high (indicated by the black bars with increasing width). The matrix is filled by comparing all the data points from both sets. Light grey indicates -1 corresponding to the case $\mathrm{DH}>\mathrm{p} 63 \mathrm{RhoGEF}$ and dark grey indicates +1 when $\mathrm{DH}<$ p 63RhoGEF.

(C) A graph that shows the resulting Cliff's delta (-0.32) and the error bars indicate the $95 \%$ confidence interval $[-0.05,-0.55]$. 


\section{Conclusion}

To conclude, I report on a VBA macro implemented in Excel for calculating Cliff's delta and its $95 \%$ confidence interval for a two-group randomized experiment. This tool should lower the barrier for calculating effect sizes in basic research and it can be used for teaching to explain the calculation of Cliff's delta.

\section{Acknowledgments}

I would like to thank Paul Goedhart (Wageningen UR, The Netherlands) for comments and Marten Postma (University of Amsterdam, The Netherlands) for explaining statistical concepts and enlightening discussions.

\section{Competing interests}

The author declares no competing or financial interests.

\section{References}

Claridge-Chang, A., and Assam, P. N. (2016). Estimation statistics should replace significance testing. Nat. Methods 13, 108-109.

Cliff, N. (1993). Dominance statistics: Ordinal analyses to answer ordinal questions. Psychol. Bull. 114, 494-509.

Cliff, N. (1996). Answering Ordinal Questions with Ordinal Data Using Ordinal Statistics. Multivariate Behav. Res. 31, 331-350.

Cumming, G. (2014). The new statistics: Why and how. Psychol. Sci. 25, 7-29.

Delaney, H. D., and Vargha, A. (2002). Comparing several robust tests of stochastic equality with ordinally scaled variables and small to moderate sized samples. Psychol. Methods 7, 485-503.

Drummond, G. B., and Tom, B. D. M. (2012). Presenting data: Can you follow a recipe? Br. J. Pharmacol. 165, 777-781.

Feng, D., and Cliff, N. (2004). Monte Carlo Evaluation of Ordinal d with Improved Confidence Interval. J. Mod. Appl. Stat. Methods 3, 322-332.

Goodman, S. (2008). A Dirty Dozen: Twelve P-Value Misconceptions. Semin. Hematol. 45, 135-140.

Halsey, L. G., Curran-Everett, D., Vowler, S. L., and Drummond, G. B. (2015). The fickle P 
value generates irreproducible results. Nat. Methods 12, 179-185.

Hsu, L. M. (2004). Biases of success rate differences shown in binomial effect size displays. Psychol. Methods 9, 183-197.

Ivarsson, A., Andersen, M. B., Stenling, A., Johnson, U., and Lindwall, M. (2015). Things we still haven't learned (so far). J. Sport Exerc. Psychol. 37, 449-461.

Li, J. C.-H. (2015). Effect size measures in a two-independent-samples case with nonnormal and nonhomogeneous data. Behav. Res. Methods, 1-15.

Motulsky, H. J. (2015). Common misconceptions about data analysis and statistics. Br. J. Pharmacol. 172, 2126-2132.

Nakagawa, S., and Cuthill, I. C. (2007). Effect size, confidence interval and statistical significance: a practical guide for biologists. Biol. Rev. Camb. Philos. Soc. 82, 591605.

Nuzzo, R. (2014). Scientific method: statistical errors. Nature 506, 150-152.

Ruscio, J. (2008). A probability-based measure of effect size: robustness to base rates and other factors. Psychol. Methods 13, 19-30.

Schneider, J. W. (2015). Null hypothesis significance tests. A mix-up of two different theories: The basis for widespread confusion and numerous misinterpretations. Scientometrics 102, 411-432.

Spitzer, M., Wildenhain, J., Rappsilber, J., and Tyers, M. (2014). BoxPlotR: a web tool for generation of box plots. Nat. Methods 11, 121-122.

Sullivan, G. M., and Feinn, R. (2012). Using Effect Size-or Why the P Value Is Not Enough. J. Grad. Med. Educ. 4, 279-282.

Tressoldi, P. E., Giofré, D., Sella, F., and Cumming, G. (2013). High impact = high statistical standards? Not necessarily so. PLoS One 8, e56180.

van Unen, J., Reinhard, N., Yin, T., Wu, Y., Postma, M., Gadella, T., and Goedhart, J. (2015). Plasma membrane restricted RhoGEF activity is sufficient for RhoA-mediated actin polymerization. Sci. Rep. 5, 14693.

Vargha, A., and Delaney, H. D. (2000). A Critique and Improvement of the CL Common Language Effect Size Statistics of McGraw and Wong. J. Educ. Behav. Stat. 25, 101132.

Wasserstein, R. L., and Lazar, N. A. (2016). The ASA's statement on p-values: context, process, and purpose. Am. Stat., posted online: 07 Mar 2016. 
bioRxiv preprint doi: https://doi.org/10.1101/073999; this version posted September 10, 2016. The copyright holder for this preprint (which was not certified by peer review) is the author/funder, who has granted bioRxiv a license to display the preprint in perpetuity. It is made available under aCC-BY 4.0 International license.

\section{Supplemental Material}

Text-macro-160905.txt

VBA macro to calculate Cliff's delta and $\mathrm{Cl}$ and dominance matrix .

Excel-CLIFFS_DELTA_160905.xIsm

An Excel workbook with the macro and data presented in figure 1. 